\title{
Pandemic (COVID-19) Killing Thousands by the Hour-What the Community Need to Know
}

\author{
Corresponding Author Naaz Ikram \\ Karachi Institute of Medical Sciences (KIMS), Affiliated with National University of Medical Sciences \\ (NUMS), Malir Cantonment, Karachi Pakistan \\ Hamayun Shahbaz \\ Karachi Institute of Medical Sciences (KIMS), Affiliated with National University of Medical Sciences \\ (NUMS), Malir Cantonment, Karachi
}

\begin{abstract}
Since the first reports of novel Pneumonia (COVID-19) in Wuhan, Hubei province, China, there has been considerable discussion on the origin of the causative virus, SARS-CoV-23 (also referred to as HCoV-19) (Wu, F. et al.2020). Infections with SARS-CoV-2 are now widespread, and as of 11 March 2020, 121,564 incidents have been verified in more than 110 nations, with 4,373 deaths. SARS-CoV-2 is the seventh coronavirus known to affect human being; SARS-CoV, MERS CoV and SARS-CoV-2 can cause severe disease, whereas HKU1, NL63, OC43 and 229E are linked with mild symptoms Purpose: The aim of this study is to gather current literature whilst bringing together the relevant information in a sequential order to identify the routes the virus takes to spread amongst the communities, along with the preventive measure that can be taken to break the chain of spread. Implications: This paper intends to provide a bird eye view on the review of the literature on perspective on the notable features of the SARS-CoV-2 genome and discuss scenarios by which they could have arisen.
\end{abstract}

Keywords: COVID-19, Pandemic

DOI: $10.7176 / \mathrm{JHMN} / 73-12$

Publication date: April $30^{\text {th }} 2020$

Background: In Wuhan, China, late during December 2019, patients exhibited with viral pneumonia due to a mysteriously anonymous microbial agent. A Virus, provisionally named 2019 novel coronavirus (2019-nCoV), was later found as the causative pathogen. As of Jan 26, 2020, more than 2000 cases of 2019-nCoV infection have been confirmed, a myriad of which involved subjects being residents of Wuhan or recent visits to the place (Roujian, Lu et al 2020). Scientists believe that the virus may have initially developed in bats, and later in pangolins (Shield,2020). Genomic comparisons, however, advocate that the SARS-Cov-2 virus is a recombination of two different viruses, putting forth the fact the virus's origin is still unclear (Alexandre Hassanin,2020).

Even though initially the virus was limited to an open seafood and livestock market in the city of Wuhan, its outbreaks now involve large numbers of people in South Korea, Iran and Italy. Local transmission is also occurring on a smaller scale in other countries. Travel restrictions and quarantine measures have been placed in some densely infected areas. Even though the number of new cases reporting in the area of its initial origin, namely China, are declining, the scope and trajectory of infection elsewhere remains to be seen (Hessen,2020)

What is CoV: Coronaviruses (CoV) have been recognized as human pathogens since the 1960's as well as pathogens to many other vertebrates. Morphology, Structure and Replication Coronaviruses are so named because of their characteristic solar corona (crown-like) appearance when observed under an electron microscope. This form is generated by the peplomers of the spike [S] glycoprotein radiating from the virus lipid envelope (Chan, J.F. et al (2015). Mainly two envelope proteins are present. (Song, Z. et al (2019) described that S glycoprotein is a main antigen in control for both receptor binding and cell fusion moreover another transmembrane glycoprotein $[\mathrm{M}]$ is included in budding and envelope development; (Tseng, Y.T. et al (2010) states that the M protein plays a vital role in the formation of virion assembly. Some of the coronavirus species includes a 3rd glycoprotein, which is known as the haemagglutinin-esterase [HE]. The viral genome is linked with the main phosphoprotein [N] inside the capsid. The genome is non segmented, positive single-stranded RNA of about 26-32 kb, making it the longest RNA viral genome known, and contains from 7 to 10 different open reading frames. The RNA molecule has a methylated cap in 5' and a poly-A tail in 3' (Kilianski, A. et al (2014); Song, Z. et al (2019) Coronaviruses can adapt quickly to new hosts via the procedures of genetic recombination and mutation in vivo. As RNA viruses, coronaviruses rely on RNA-dependent RNA 5 polymerase $(\mathrm{RdRp})$ to imitate the virus genome. The intrinsic error rate of $\mathrm{RdRp}$ is around 1,000,000 mutation/site/replication, causing in uninterrupted point mutations. Point mutations alone are not enough to generate a new virus, nevertheless; this can only occur when the same host is concurrently infested with two coronavirus strains, enabling recombination. One coronavirus can gain a genomic fragment of hundreds or thousands base-pair long from another CoV strain when the two co-infect the same host, enabling the virus to increase its ecological niche or to make the leap to a new species (Chan, P.K. et al (2013); Raj, V.S. et al (2014); Gralinski, L.E. et al (2015)). This vulnerability allowed the emergence in nearly two decades 
of three new human coronavirus species with epidemic potential: SARS-CoV, MERSCoV and 2019-nCoV.

History of its Spread in Humans: In 2012, MERS, a corona virus that had the capability of causing a severe acute respiratory illness, emerged in humans in Saudi Arabia. Other cases continued to be reported in other parts of the world as well within the course of one year namely in France, Germany, Jordan, Qatar, Tunisia, the United Arab Emirates, and the United Kingdom. All confirmed cases were directly or indirectly connected to the Middle East. Of all verified cases documented by 2019 , approximately one-third ended in death (Song Z, Xu Y, Bao L, et al.2019). The novel MERS coronavirus shared a similarity with others of its kind in that it also originated in bats, later being transmitted to humans, but, in its specific case, camels were recognized as one possible reservoir for it. In late 2019, in Wuhan, china, a virus seemingly closely related to SARS coronavirus emerged. The Wuhan coronavirus, later named severe acute respiratory syndrome coronavirus 2 (SARS-CoV-2), caused an illness known as COVID-19, which was like SARS and was being portrayed primarily by fever and respiratory symptoms. The virus was also extremely contagious. Just in a matter of a month, it had dispersed to infect thousands in Europe and united states by carriers of the viral disease traveling from affected areas to uninfected ones. In March, just 2 months later to the initial spread, WHO declared the outbreak as Pandemic.

How does the virus spread and what are the symptoms? The WHO reports that human-to-human spread is taking place with an initial R0 estimate of 1.4-2.5. Recent assessments of the incubation period of the COVID-19 range from two to fourteen days, and it appears to be spread primarily via flu-like signs and respiratory droplets that human beings sneeze, cough, or breathe out. Normal signs of COVID-19 involve fever, cough, trouble breathing, muscle pain and fatigue. More severe cases develop severe pneumonia, acute respiratory distress syndrome, sepsis and septic shock. Normally, elderly and those with underlying conditions (such as hypertension, heart disorders, diabetes, liver disorders, and respiratory disease) are likely to be more at risk of developing severe symptoms (Zhou, et al 2020). The evidence from analyses of cases to date is that COVID-19 infection causes mild disease (i.e. non-pneumonia or mild pneumonia) in about $80 \%$ of cases and most cases recover; $14 \%$ have more severe disease and $6 \%$ experience critical illness.

Impact of COVID-19 on main organs: Lungs: Ground zero: For majority of the patients, COVID-19 commences and stops in their lungs, because like the flu, coronaviruses are respiratory diseases. The symptoms of the patient might begin with fever and cough but may develop into pneumonia or even worse. After the SARS outbreak, the World Health Organization reported that the disease usually hit the lungs in three phases: viral replication, immune hyper-reactivity, and pulmonary destruction. However, not all the patients went through all three of them. In fact, only one fourth of the patients went through a respiratory failure, a symptom which is thought to be a signature for the disease. Similarly, COVID-19, according to early data, causes severe symptoms in only $12 \%$ the patients, while the remainder show mild symptoms only Look deeper, and the novel coronavirus seems to follow other patterns of SARS, says University of Maryland School of Medicine associate professor Matthew B. Fireman, In its early days, the novel corona virus affects mainly two types of cells in the lungs; the mucus cells which produce mucus which aids protection of lung tissue from pathogens and makes sure your breathing organ doesn't dry out, and the cilia cells which have hair like structures on the surface of cells that brush and beat to clear out debris from the respiratory airway. The earliest studies on covid 19 have shown that the patients in phase 1 develop pneumonia in both the lungs. This is important to note down as this is where phase 2 of the disease starts to hit the lungs. The immune system starts to fight of the disease by recruiting lots of immune cells to the infected area to resolve the state of the lungs, but since the immune system has gone haywire, the immune cells kill anything in their sight causing more damage than actual help. Thus, Even more debris clogs up the lungs, and pneumonia aggravates. Upon the beginning of the 3rd phase, more lung damage starts to occur, progressing towards respiratory failure and permanent lung damage. According to the WHO World Health Organization, SARS and the covid-19 caused the lungs to resemble a "honeycomb" like appearance due the presence of holes, created by the immune system's hyperactive response. These holes are meant to protect and stiffen the lungs after they are formed into scars. This phase is when the patients are in desperate need of ventilator to aid in their breathing. Parallel to the lung damage, continuous inflammation makes the membranes between the air sacs and blood vessels more permeable, which can fill the lungs with fluid and disturb their capability to oxygenate blood. "In severe cases, you basically flood your lungs and you can't breathe," Frieman says. "That's how people are dying." (ED, Young, 2020)

The Stomach: A mutual gateway: During a corona virus outbreaks, a common shared feature or symptom among the patients was diarrhea accompanied with abdominal pain. The question, however, is: what does a respiratory virus have to do with the gut? The answer is that the virus attaches to any receptor on a cell it can find and is compatible with and penetrates it to cause damage. They can very easily penetrate into all types of cells," says Anna Suk-Fong Lok, assistant dean for clinical research at the University of Michigan Medical School and former president of the American Association for the Study of Liver Diseases. Does that mean it can spread through feces too? Two studies - one in the New England Journal of Medicine and one preprint in medRxiv? involving 1,099 cases - have also detected the virus in stool samples, which could indicate the virus could spread via feces. This information however is far from being conclusive. "Whether that kind of fecal transmission is occurring for this 
Wuhan virus, we don't know at all," Frieman says. "But it definitely looks like it's there in the stool and it looks like people do have GI symptoms associated with this."

Liver: Collateral devastation: The virus is carried to the liver via bloodstream where it damages the liver cells and causing their death. The death of these cells releases enzymes into the blood stream. The more the damage, the higher the levels of enzymes in the blood. This is demonstrated by patients suffering from SARS and MERS. The patients with the corona virus show abnormally high levels of liver enzymes in their blood. Lok says scientists still don't completely comprehend how these respiratory viruses work in the liver. Either way, she notes that liver failure was certainly not the single cause of death for any of the SARS patients. "By the time the liver fails," she says, "often you'll find that the patient not only has lung problems and liver problems, but they may also have kidney problems. By then it becomes a systemic infection."

Kidney: It's all connected: $6 \%$ of SARS patients and 75\% of MERS patients suffered acute renal injury according to a recent study. Studies have also shown that the novel corona virus can present with the same level of damage even though it is rare it does so. Eventually 91.7 percent of SARS patients with acute renal impairment died, according to a 2005 study in Kidney International. After the SARS outbreak, the WHO reported that the virus was found in kidney tubules, which can become inflamed. This information inferred that it Is the kidney tubules that showed the most damage. "It's not uncommon to detect a virus in the tubules if it's in your bloodstream", says Kar Neng Lai, a professor emeritus at the University of Hong Kong and consultant nephrologist at Hong Kong Sanatorium and Hospital. As your kidneys are constantly filtering blood, every so often the tubular cells can trap the virus and cause a temporary, or milder, injury. The injury, however, could become lethal if the virus penetrates the cell and starts to replicate itself within it. But Lai-who was also a member of the first group of researchers writing on SARS and added to the Kidney International study_-says there was no evidence that the SARS virus was replicating in the kidney. That finding, Lai says, implies acute kidney injury in SARS patients may possibly be due to a diverse set of triggers, including low blood pressure, sepsis, drugs, or a metabolic disturbance. Other triggers include antibiotics, multiorgan failure, or long-term ventilator usage.

Multi system involvement: bloodstream: Due to the activation of a hyperactive immune response, coronavirus can involve several other organs and organ systems as well. A study in 2014 portrayed that $92 \%$ of the patients with MERS showed at least one manifestation of the coronavirus other than that of the lungs. In fact, signs of an all system involvement have been observed with all three of the zoonotic coronaviruses: elevated liver enzymes, lower white blood cell and platelet count, and low blood pressure. patients, however, have also suffered from acute kidney injury and cardiac arrest, in rare cases. "But this isn't necessarily a sign that the virus itself is spreading throughout the body", says Angela Rasmussen, a virologist and associate research scientist at Columbia University Mailman School of Public Health. Thus, implying that the damage might be related to an abnormally increased levels of cytokines. Cytokines are proteins utilized by the immune system as alarm signs - they hire immune cells to the site of infection. The infected tissue is then destroyed by the immune cells to save to remainder of the noninfected body. "During a runaway coronavirus infection, when the immune system dumps cytokines into the lungs without any regulation, this culling becomes a free-for-all" Rasmussen says, "Instead of shooting at a target with a gun, you're using a missile launcher," she says. That's where a conflict surfaces: the body is not just targeting infected cells; it is also destroying the body's healthy tissues too. This conflict spreads outside the lungs. Blood vessels of the lungs are weakened and fluid seeps through to the air sacs. "Basically, you're bleeding out of your blood vessels," Rasmussen says. The cytokines continue their destruction into the circulatory system and creates systemic issues across a myriad of organs. This is the part where things begin to get worse. The cytokine response plus the diminished capacity to pump oxygen to the rest of the body result in multi organ failure in some very severe cases of the COVID-19.Underlying conditions like heart disease or diabetes are shown to be linked to complications outside of the lungs. "Even if the virus doesn't get to kidneys and liver and spleen and other things, it can have clear downstream effects on all of those processes,"

So how can we break the chain? Infection prevention and control in the population the usage of individual protective measures (i.e. rigorous hand hygiene, cough etiquette, and face masks) may add to lowering the risk of spreading or developing COVID-19 infections. Rigorous hand-washing methods, involving washing of hands with soap and water for at minimum 20 seconds, or scrubbing hands with alcohol-based solutions, gels or tissues is advised in all public venues in all likely circumstances. Companies should safeguard accessibility of adequately and appropriately located sinks and taps, as well as hand gels, to promote washing. Appropriate hand hygiene will also diminish the spread of other contagious diseases (ECDC, 2020). Shielding the mouth and nose while coughing and sneezing (e.g. by utilizing a paper tissue, and sneezing or coughing into the elbow) may automatically block the drop spread that is the principal spread mode for COVID-19. The correct disposal of used tissues is vital, followed by instant washing of hands coughing/sneezing. The usage of surgical face masks reduces the risk of infecting others when worn by a human with respiratory symptoms prior to obtaining medical advice and while being evaluated. There is no evidence on the effectiveness of face masks worn by persons who are not ill, therefore this is not advisable (ECDC, 2020). It is a possibility that the usage of facemasks by inexperienced individuals may even expand the risk of contagion due to a false sense of protection and intensified contact between hands, 
mouth and eyes. Moreover, Personal social distancing measures (e.g. avoiding shaking hands and kissing and avoiding crowded carriages and unnecessary gatherings and meetings) should be advised at organizational, national levels as a precautionary measure. Further steps incorporate school and day care procedures or closings, actions at the office, and measures linked to mass gatherings. In some countries such as China, and more recently in Italy, internal travel restrictions or "Cordon sanitaire" have been imposed on large populations together with other containment measures. The evidence for the effectiveness of closing schools and workplaces, and cancelling mass gatherings is limited. However, one investigation from China projected that if a range of non-pharmaceutical interferences, including social distancing, had been conducted one week, two weeks, or three weeks earlier in the country, the number of COVID-19 cases could have been reduced by $66 \%, 86 \%$, and $95 \%$, correspondingly, together with significantly reducing the number of affected areas (Lai S, Ruktanonchai NW, Zhou L, Prosper O, Luo W, Floyd JR, et al,2020). Data originating from seasonal and pandemic influenza modelling experiments have demonstrated that proactive school closings before the peak of influenza virus activity have had a positive influence in decreasing local transmission and delaying the peak of the influenza activity (World Health Organization (WHO,2019).

\section{References}

Alexandre Hassanin, 2020 https:/www.weforum.org/agenda/authors/alexandre-hassanin

C. L. Paules, H. D. Marston and A. S. Fauci. Coronavirus infections - more than just the common cold. JAMA. January 23, 2020. doi:10.1001/jama.2020.0757.

Chan, J.F., Lau, S.K., To, K.K., Cheng, V.C., Woo, P.C., Yuen, K.Y. Middle East respiratory syndrome coronavirus: another zoonotic beta coronavirus causing SARS-like disease. Clin Microbiol Rev 2015, 28(2): 465.

Channappanavar R, Perlman S. Pathogenic human coronavirus infections causes and consequences of cytokine storm and immunopathology. Seminars in Immunopathology. 2017 July 01;39(5):529-39.

Charli Shield,2020 https://p.dw.com/p/3XPQQ Accessed on 20/03/2020

Chen N, Zhou M, Dong X, et al. Epidemiological and clinical characteristics of 99 cases of 2019 novel coronavirus pneumonia in Wuhan, China: a descriptive study. Lancet 2020; 395: 507-13.

Danese, S., Cecconi, M. \& Spinelli, A. Management of IBD during the COVID-19 outbreak: resetting clinical priorities. Nat Rev Gastroenterol Hepatol (2020). https://doi.org/10.1038/s41575-020-0294-8

ED YONG MARCH 20, 2020 https://www.theatlantic.com/science/archive/2020/03/biography-newcoronavirus/608338/ Accessed at 02/04/2020

European Centre for Disease Prevention and Control. Novel coronavirus disease 2019 (COVID-19) pandemic: increased transmission in the EU/EEA and the UK - sixth update - 12 March 2020. Stockholm: ECDC; 2020.

Fei Zhou* TY, Ronghui Du*, Guohui Fan*, Ying Liu*, Zhibo Liu*, Jie Xiang*, Yeming Wang, Bin Song, Xiaoying Gu, Lulu Guan, Yuan Wei, Hui Li, Xudong Wu, Jiuyang Xu, Shengjin Tu, Yi Zhang, Hua Chen, Bin Cao,. Clinical course and risk factors for mortality of adult inpatients with COVID-19 in Wuhan, China: a retrospective cohort study. The Lancet. 2020 March 9, 2020.

https://www.sciencenewsforstudents.org/article/explainer-what-is-a-coronavirus

Kara Rogers,2020https://www.britannica.com/science/coronavirus-virus-group

Kilianski, A., Baker, S.C. Cell-based antiviral screening against coronaviruses: Developing virus specific and broad-spectrum inhibitors. Antiviral Res 2014, 101: 105.

Lai S, Ruktanonchai NW, Zhou L, Prosper O, Luo W, Floyd JR, et al. Effect of non-pharmaceutical interventions for containing the COVID-19 outbreak: an observational and modelling study. medRxiv. 2020:2020.03.03.20029843.

Lu, Roujian \& Zhao, Xiang \& Li, Juan \& Niu, Peihua \& Yang, Bo \& Wu, Honglong \& Wang, Wenling \& Song, Hao \& Huang, Baoying \& Zhu, Na \& Bi, Yuhai \& Ma, Xuejun \& Zhan, Faxian \& Wang, Liang \& Hu, Tao \& Zhou, Hong \& Hu, Zhenhong \& Zhou, Weimin \& Zhao, li. (2020). Genomic characterization and epidemiology of 2019 novel coronavirus: implications for virus origins and receptor binding. The Lancet. 395. 10.1016/S0140-6736(20)30251-8.

Margaret Trexler Hessen, MD, Director, Point of Care, Elsevier https:/www.elsevier.com/connect/coronavirusinformation-center

Middle East respiratory syndrome coronavirus (MERS-CoV) (World Health Organization) http://www.who.int/emergencies/mers-cov/en/

Simon Makin,2020https://www.scientificamerican.com/article/how-coronaviruses-cause-infection-from-coldsto-deadly-pneumonial/

Song $\mathrm{Z}, \mathrm{Xu} \mathrm{Y}$, Bao $\mathrm{L}$, et al. From SARS to MERS, thrusting coronaviruses into the spotlight. Viruses. 2019;11(1):11. doi:10.3390/v11010059PubMedGoogle ScholarCrossref

Song, Z., Xu, Y., Bao, L., et al. From SARS to MERS, thrusting coronaviruses into the spotlight. Viruses 2019 , 11(1): E59. 
Tseng, Y.T., Wang, S.M., Huang, K.J., Lee, A.I., Chiang, C.C., Wang, C.T. Self-assembly of severe acute respiratory syndrome coronavirus membrane protein. J Biol Chem 2010, 285(17): 12862

University of California - Los Angeles. (2020, March 20). Study reveals how long COVID-19 remains infectious on cardboard, metal and plastic: People may acquire coronavirus through air and by touching contaminated $\begin{array}{llllll}\text { surfaces. } & \text { ScienceDaily. } & \text { Retrieved } & \text { March } & 24, & \text { from }\end{array}$ www.sciencedaily.com/releases/2020/03/200320192755.htmuniversity of California https://www.sciencedaily.com/releases/2020/03/200320192755.htm

W. Ji et al. Homologous recombination within the spike glycoprotein of the newly identified coronavirus may boost cross-species transmission from snake to human. Journal of Medical Virology. January 22, 2020. doi: 10.1002/jmv.25682.

World Health Organization (WHO). Non-pharmaceutical public health measures for mitigating the risk and impact of epidemic and pandemic influenza 2019 [cited 20201 March]. Available from: https://apps.who.int/iris/bitstream/handle/10665/329438/9789241516839-eng.pdf?ua=1 